\title{
Temperature measurements during laser tissue welding process
}

by T. Piatkowski, A. Zajac, H. Polakowski, M. Kastek

Institute of Optoelectronics, Military University of Technology, gen. S. Kaliskiego 2, 00-908 Warsaw, Poland, tpiatkowski@wat.edu.pl

\begin{abstract}
Laser welding of biological tissues require temperature control over the entire process, because the allowed temperature margin is $2{ }^{\circ} \mathrm{C}$ around the tissue-specific coagulation temperature. The paper presents the results of the research concerning laser tissue welding, using continuous wave laser diode. The infrared radiation associated with laser welding process was detected by two thermal imaging cameras. First one, operating in SWIR range, measured the scattered radiation of a laser diode. The second camera, operating in MWIR spectral band was used to measure the temperature of a tissue. The rates of tissue temperature changes, surface and in-depth temperature distributions were measured for different kinds of tissues and power outputs 20-75W of applied semiconductor laser.
\end{abstract}

\section{Introduction}

Laser welding of tissues $[1,2,3]$ is one of the surgical applications of laser devices. The aim of this process is to obtain firm bonding, which is achieved by heating the tissue to the temperature slightly above coagulation threshold for the time needed to create irreversible changes of the treated structure. The required temperature level is said to be within $2{ }^{\circ} \mathrm{C}$ margins above the tissue coagulation temperature which in case of human tissues may vary from $65^{\circ} \mathrm{C}$ to $95^{\circ} \mathrm{C}$, depending on the kind of the tissue. The main problem is then to keep the temperature of the tissue area exposed to laser radiation within acceptable margin to avoid destructive effects like vaporization, which makes the healing impossible. The main problem is then to keep the temperature of the tissue area exposed to laser radiation within acceptable margin to avoid destructive effects like vaporization, which makes the healing impossible. To achieve this, constant monitoring of the laser interaction with a tissue is required and the trouble is that each kind of a tissue has different properties, which are not usually determined $[4,5]$. As a result it is not possible to subjectively assess the actual condition of the treated tissue. Thus, a real time control and measurement procedures required monitoring the actual status of the treated tissue and then to regulate the laser parameters in a feedback loop during the whole tissue welding process.

The required feedback signal can be obtained using basically two methods. First method relies on spatial analysis of laser radiation scattered from the surface of a welded tissue [6, 7]. The source of laser radiation is usually auxiliary He-Ne laser [14]. The diagnostic signal (scattered radiation) has in this case low power and additionally its spatial distribution has to be determined. As a result the measurement setup is complicated and the synchronous detection is required, which makes this method applicable to the laboratory measurements only. The second method is based on analyzing the tissue heating by means of thermal cameras $[8,9,10]$. It requires much simpler measurement setup and due to lowered costs of thermal cameras it is not as expensive as before. The measurement setup for the monitoring of laser welding of a tissue is presented in Fig. 1 [4].

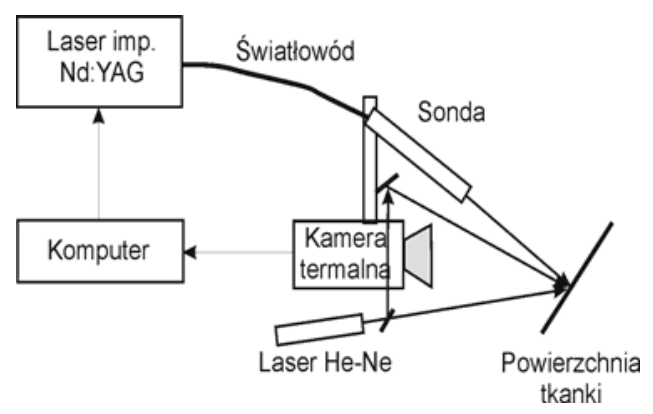

Fig 1: Measurement stand for the measurement of tissue temperature by means of a thermal camera [4].

Usually the coagulation is obtained by illuminating the tissue with pulsed Nd:YAG laser. The auxiliary He-Ne laser is used to point out the spot in which the invisible beam of a main laser (supplied by an optical fiber probe) hits the tissue. The welding procedure can be divided into three stages [5]. In the first stage a single probing pulse is generated.ith parameters (duration, power) matched to the properties of the illuminated tissue. After the pulse the thermal camera 
records the resulting temperature changes and the data are sent to the computer for analysis. In the next stage, after the analysis, the parameters of a tissue coagulation are determined (maximal temperature, minimal temperature and time span between consecutive pulses). In the final stage the computer programs the pulse sequence for the Nd:YAG laser. In this sequence the duration of the first pulse is adjusted to achieve maximal coagulation temperature. The duration of each next pulse is adjusted to keep the tissue temperature in the desired range where the coagulation occurs.

The tissue welding method described above has several disadvantages. First, three parameters of laser illumination have to be determined: pulse duration, repetition rate and pulse power. Furthermore, the selection of beam energy on the basis of surface temperature after the pilot pulse is inaccurate, and the internal tissue temperature is usually higher $[11,12,13]$ because the measurement does not take into account volumetric absorption properties of a tissue. Because of all those disadvantages the laser diode operating in $\mathrm{CW}$ regime was used in the next experiments.

\section{Tissue welding process}

Thermal damage may occur in a laser welding when the tissue is heated by a laser beam above the coagulation threshold. In the early stage of welding process the coagulated areas are glued together, but the mechanical strength of such bonding is low. The main condition that should be met to obtain proper welding is to create narrow, homogenous temperature distribution around the welding spot. In practice this condition is rarely fulfilled, because due to absorption much less laser energy penetrates the deeper layers of a tissue and consequently those internal areas are less heated. Furthermore, about $15 \%$ of energy is absorbed outside the geometrical boundaries of a laser beam due to scattering and large portion of energy could also be reflected.

By analyzing the methods used to assess the tissue condition during laser welding it was attempted to correlate the results given by optical and thermal imaging methods. There are two reasons to consider the thermal imaging method - the thermal cameras are becoming more affordable and offer increased functionality. The temperature distributions were measured for a chosen tissue at different power settings of a working laser. During the experiments the semiconductor laser was used with a power output up to $90 \mathrm{~W}$, operating at a wavelength $\lambda=980 \mathrm{~nm}$. The laser was coupled with an optic fiber which resulted in beam configuration similar to probes used for medical procedures. The laser diode (LD) was composed of an array of emitters and as a result a uniform power density distribution was obtained in transversal plane in spite of an overall low beam quality ( $\mathrm{M}^{2}$ quality factor was around 100$)$. The tissue area exposed to laser radiation was inspected using thermal cameras.

\section{Stand for tissue welding process}

The block diagram of a laboratory measurement stand is shown in Fig. 2a. Semiconductor laser is coupled with an optic fiber, whose other end is fixed by a grip. The grip can be precisely regulated to assure the proper positioning of an optic fiber with respect to the lens. The laser beam is focused by the lens on the tissue sample, which is placed on a $\mathrm{XYZ}$ table for a fine positioning in space.

a)

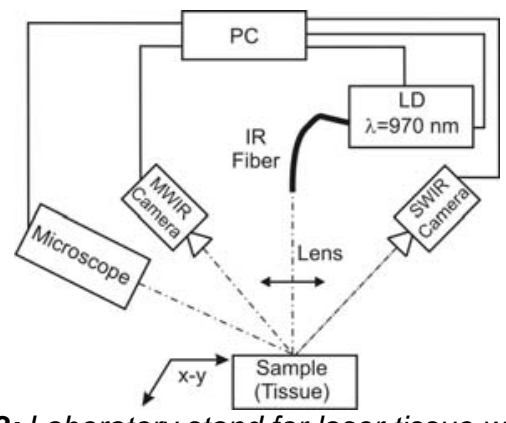

b)

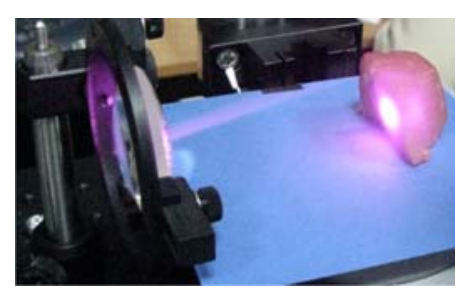

Fig 2: Laboratory stand for laser tissue welding: a) block diagram b) photo of an exposed tissue sample

The sample area illuminated by a laser beam was viewed by two IR cameras. First camera is a measurementclass mid wave infrared (MWIR) camera, operating in a spectral range from $3 \mu \mathrm{m}$ to $5 \mu \mathrm{m}$. It has cooled InSb focal plane array with a resolution of $640 \times 512$ pixels. This camera is totally insensitive to the used laser wavelength but due to its working range and chemical composition of tissue sample $(30-90 \%$ of water) it can measure the surface temperature distribution. Second camera is a short wave infrared (SWIR) observation device, covering the spectral range from $0.7 \mu \mathrm{m}$ to $2.5 \mu \mathrm{m}$. It has an array MCT detector, $320 \times 256$ pixel resolutions, cooled by thermoelectric cooler. The SWIR camera was used because it is sensitive to the wavelength of the applied laser, thus it should be possible to visualize the laser radiation scattered from the tissue. High power output of the applied laser provided detectable and sometimes even too big signal. Contrary to the detection of scattered radiation of He-Ne laser, in this case some no special measurement 
procedures are required $[6,10]$. Furthermore the radiation in SWIR range is less attenuated then MWIR, so it might be possible to record the signal from deeper tissue layers. The integration time and f-number of camera lens was adjusted to detect just that signal. It turned out that the power of scattered laser radiation was too big and local detector saturation was observed. To prevent this cut-off filter was applied with $1.1 \mu \mathrm{m}$ limit. Another reason to use SWIR camera was many of different types of array detectors working in this particular spectral range (like InGaAs) which translates into possible optimization of final device and increase in measurement accuracy.

The test stand was also equipped with a microscope coupled with a CCD camera and digital still camera for the visual imagery of treated tissue samples. Sample photo taken during measurement session is shown in Fig. 2b. The scattered laser radiation was recorded even though the wavelength of the applied laser is beyond visible range.

Two series of measurements were conduced in order to determine the relation between exposure time and resulting thermal effects inside the tissue volume, temperature distributions and magnitude of scattered radiation. In the first series of measurements the focal point of a laser beam was located just beneath the surface of a tissue sample. It was measured (by slicing the sample after the measurements) that the actual depth was: $L=0.5 \mathrm{~mm}(+0.3 \mathrm{~mm})$. These measurements were conducted to determine how deep the laser beam penetrated the tissue and also to determine the surface temperature distribution and spatial distribution of scattered laser light. Both thermal cameras were used during this measurement session. During the second measurement session the beam was focused deeper inside the tissue sample and in this case the temperature distribution was less affected by additional factors like local thermal bridges, which are likely to appear in thin layers. The laser operated in CW regime during both measurement sessions, thus the synchronization between laser and cameras was not required. The CW working regime will also be applied in a planned laser-based tissue welding device, resulting in simpler design and operation. The only user-selectable parameters will be output power and relative location of welded area, which are intuitive and easy to comprehend for an operator. The tissue was illuminated as long as tissue damage occurred (explosion in first series and carbonization in a second one).

Even though MWIR camera is insensitive to laser radiation, yet its image was disturbed by secondary heat sources - elements of measurement stand heated up by laser radiation. Such thermal effects are usually slow, but it was noticeable due to high output power of a laser, up to $90 \mathrm{~W}$. Because of that the frame rate was increased to $200 \mathrm{~Hz}$ and windowed mode $(320 \times 256$ pixels) was used. Reduced camera resolution has no negative impact on spatial resolution of the recorded images, because even that smaller field of view was large enough to cover the entire sample area.

\section{Investigation of the interactions of laser beam with bological tisues}

During the first series of measurements the temperature rise rate was determined and the laser beam-tissue interaction area was measured, including the size of damaged tissue as a function of power output of a laser. The temperature rise curves for selected spots on a muscular tissue are shown in Fig. 3. Different initial temperatures resulted from the process of sample preparation. It can be observed on the temperature plots shown in Fig. 3a.

a)

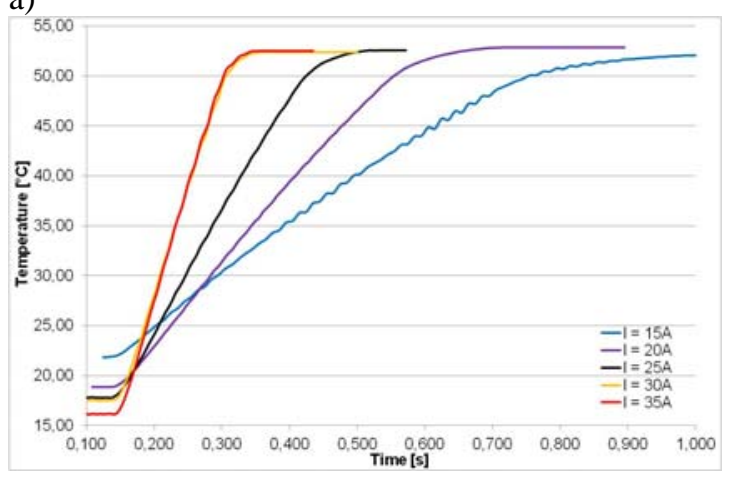

b)

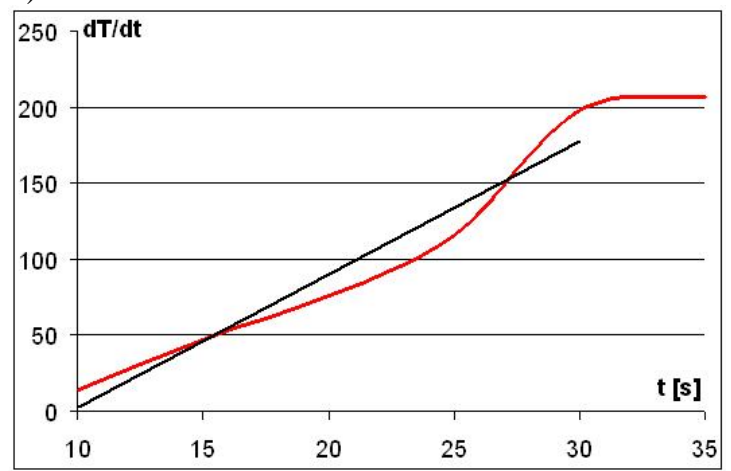

Fig 3: Temperature changes: a) rise versus time for different output power, b) rise rate as a function of output power

It can be also seen that the final temperature is more or less identical for all samples $T=53.0^{\circ} \mathrm{C} \pm 1.0^{\circ} \mathrm{C}$, regardless of initial sample temperature and laser power. This temperature results from boundary conditions for the heat transfer to the surface of a sample.

Another important parameter is the temperature rise rate as a function of laser output power. The converted data, in which the initial temperatures were taken into account, are shown in Fig. 3b. The relevant numerical data for parallel and transversal orientation of muscle fibers in tested sample with respect to the laser beam are given in Table 1. 
Table 1 Temperature rise rate as a function of laser output power

\begin{tabular}{|c|c|c|}
\hline LD power [W] & Fibers perpendicular dT/dt $\left[\mathrm{K} \mathrm{s}^{-1}\right]$ & Fibers parallel dT/dt $\left[\mathrm{K} \mathrm{s}^{-1}\right]$ \\
\hline 20 & 14,47 & 36,89 \\
\hline 30 & 47,00 & 80,04 \\
\hline 48 & 76,08 & 99,43 \\
\hline 58 & 115,30 & 108,52 \\
\hline 66 & 198,74 & 101,86 \\
\hline 75 & 207,26 & 112,23 \\
\hline
\end{tabular}

For both orientations of fibers in the tested sample the temperature rise rate is proportional to the output power, up to the power setting of $70 \mathrm{~W}$. The linear approximation of the measured relation up to this power value is shown in Fig $3 \mathrm{~b}$. for higher power settings the relation becomes strongly non-linear, with saturation area. In other words the usable range of available output power is up to $70 \mathrm{~W}$. After the illumination the size of coagulation area was determined. The photo of a test sample is shown in Fig. 4a whereas the Fig.4.b presents the measured diameters of thermally altered area and linear approximation of results.

a)

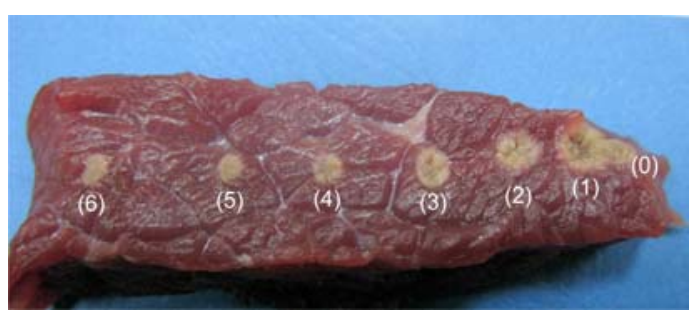

Fig 4: Coagulation diameter as a function of laser power, a) photo of the tissue sample, b) measurements and approximation line b)

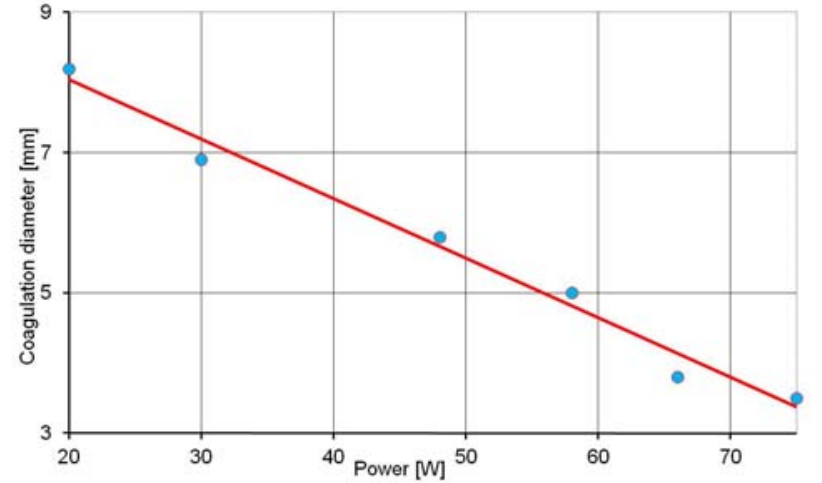

An inversely-proportional relation between the laser output power and the size of thermally altered area can be observed. It is advantageous because the necrosis area is then smaller for larger power settings, but in such situation other illumination parameters become critical, especially exposure time. The results of an experiment which ended with a carbonization of surface layer are shown in Fig.5.

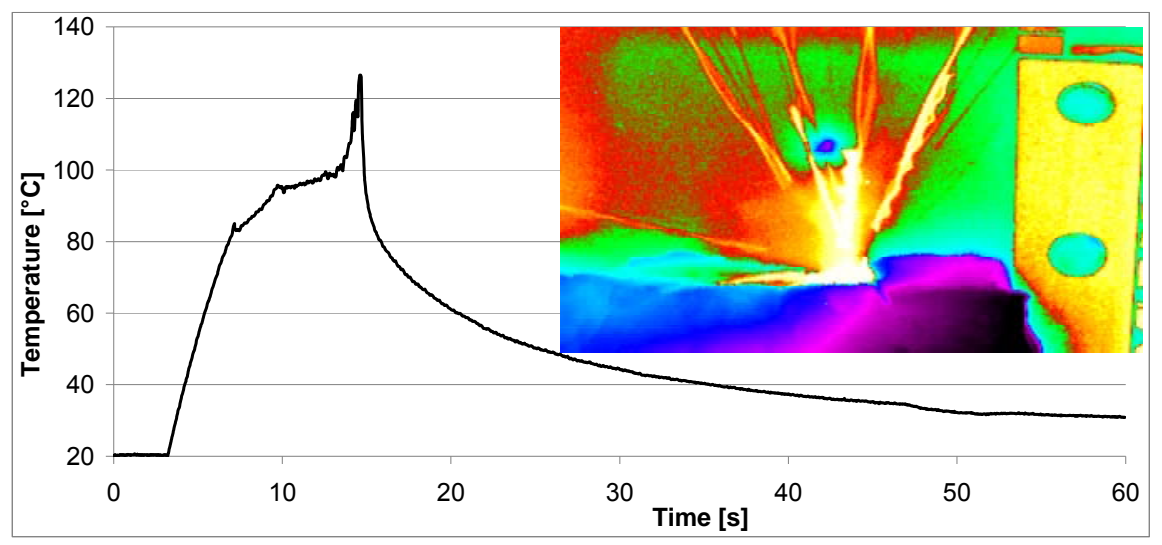

Fig 5: Temperature changes in overheated sample

The temperature has raised exponentially up to the temperatures around $85^{\circ} \mathrm{C}$. After that the temperature rose abruptly because the volumetric heating changes into surface heating. Both absorption and heat transfer conditions changed due to carbonization of the surface layer, which is reflected in a curve slope change. At the temperature of about 
$95^{\circ} \mathrm{C}$ the rapid water vaporization took place and the slope of the curve changes again. Above $100^{\circ} \mathrm{C}$ the tissue was shattered by an explosion of boiling tissue fluids. This time instant is shown on a picture above the temperature plot (Fig.5). Fast, explosive tissue damage was observed for the laser power above 60W. For such powers the image acquisition rate was hen increased to $200 \mathrm{~Hz}$.

Thermal effects observed in tissues strongly depend on the kind of a tissue itself. For many tissue types the optic and thermal properties are anisotropic. It is a reason why the temperature distribution for muscular tissues were recorded taking into account the propagation of an incident laser beam with respect to the direction of muscle fibers. For comparison a quasi-isotropic tissue was tested, represented by a liver fragment. Two parameters were analyzed during the processing of thermal image data [15] - the size of thermally altered area as a function of illumination time and the temperature changes at the selected measurement points. The area size was calculated as a number of pixels with temperatures above certain threshold. The size of thermally altered area for the muscle tissue with fibers parallel and perpendicular to the laser beam are presented in Fig. 6.

a)

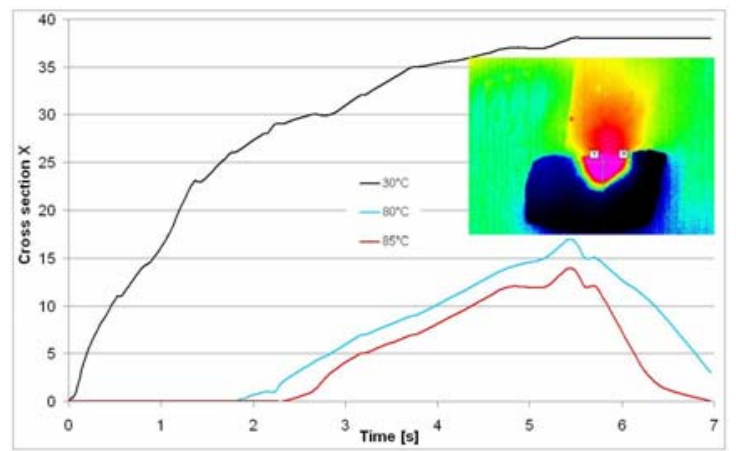

b)

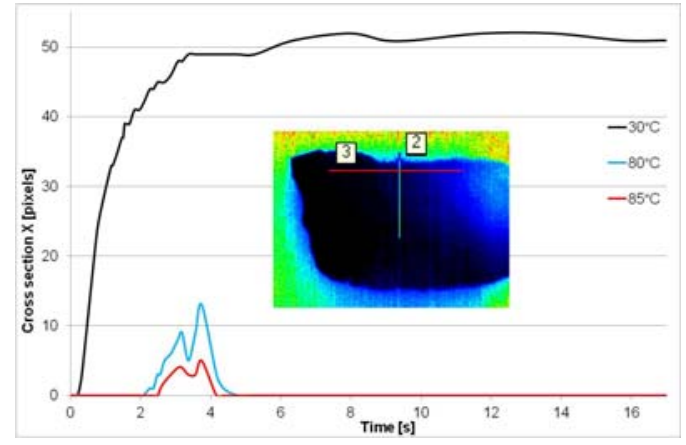

Fig 6: Number of pixels with the temperatures above given level in a muscle sample (X cross-section) for fibers a) perpendicular and b) parallel to the laser beam

In case of perpendicular fibers (Fig 6a) the affected area is smaller than those observed for parallel fibers. However, the hottest area size (pixel count) is quite comparable, because the laser-tissue interaction area is similar in both cases. The penetration depth of laser beam is significantly different, though.

Similar measurements were performed for a liver sample. In this case there are no anisotropic properties so the mutual orientation of a laser beam and a sample in not important. The size of heated areas in $\mathrm{X}$ cross section is shown in Fig. 7.

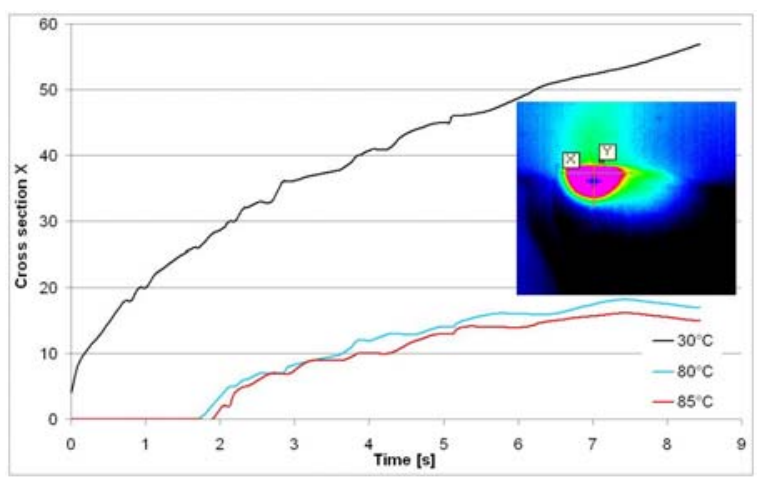

Fig 7: Number of pixels with the temperatures above given level in a liver sample (X cross-section

For a liver sample almost proportional relation between the size of the affected area and delivered laser energy. Due to isotropic properties of the liver tissue, the additional measurement of scattered laser radiation (wavelength $\lambda=970 \mathrm{~nm}$ ) was performed by a SWIR camera. The data recorded by both cameras for a chosen measurement point are depicted in Fig. 8. 


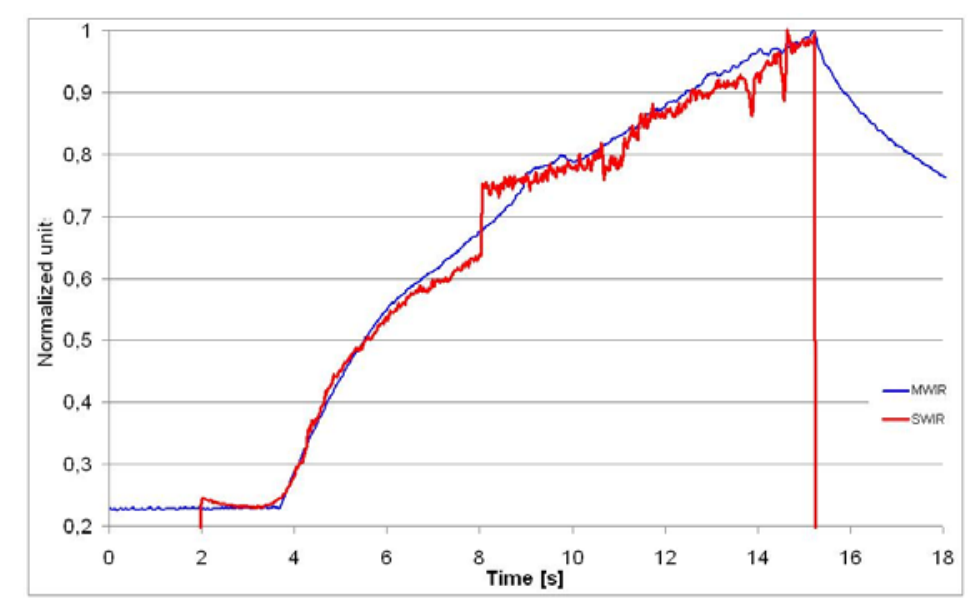

Fig 8: IR signals measured in two spectral ranges in a chosen point

The data recorded by both cameras are in different formats. MWIR camera gives temperature measurements (in degrees Celsius) whereas for SWIR camera the digital values at the output of A/D converter are recorded. In order to compare those output data, both were normalized. The $Y$ axis was limited to the range $0.2-1.0$, which, in case of SWIR camera narrows the plot only to the case when the scattered laser radiation is observed. Vertical lines in time instants of 2.0 and 15.8 seconds indicate moments of laser switching on and off, respectively. Both curves are similar up to the time instant of 8.5 second, when the level of scattered laser radiation rises considerably. This may be caused by a coagulationrelated change of scattering coefficient. The same effect was demonstrated for a He-Ne laser, which was described in [6]. The exact nature of the aforementioned effect, however, is yet to be confirmed.

\section{Conclusions}

The presented results of research on the heating of biological tissues by means of a semiconductor laser (wavelength $980 \mathrm{~nm}$, power output 20-75W) lead to the conclusion that such source of laser radiation can be applied for several medical procedures, like cutting of tissues (in a form of laser lancet) or coagulation of a tissues exposed to a laser beam. The correlation between the measurements results of coagulated area obtained by thermal imagery in different spectral bands was demonstrated. Rapid increase of the scattered laser radiation, recorded in a certain moment during coagulation by SWIR camera (not reported in previous experiments) may be exploited in medical equipment. This effect, shown in Fig. 8 occurred when the tissue reached the coagulation temperature. This correlation, described in the paper, between the magnitude of scattered radiation, surface temperature and the status of treated tissue allows for the simplified design of a laser apparatus for tissue welding.

\section{Acknowledgment}

The result presented in paper are supported by realization of the Project is co-financed by the European Regional Development Fund within the framework of the 2. priority axis of the Innovative Economy Operational Programme, 2007-2013, submeasure 2.1. "The development of centres with high research potential". Contract no. POIG.02.01.00-14-095/09.

\section{REFERENCES}

[1] Oz, M.C., Chuck, R.S., Johnson, J.P., Parangi, S., Bass, L.S., Nowygrod, R. and Treat, M.R., "Indocyanine green dye enhanced vascular welding with the nar. infrared diode laser", Vascular Surgery 24, 564-570 (1990)

[2] Small, W., "Thermal and Molecular Investigation of Laser Tissue Welding", PhD thesis Lawrence Livermore National Laboratory, (1998).

[3] Bass, L.S., Treat, M.R., "Lasertissue welding - a comprehensive review of current and future clinical applications", Laser in Surgery and Medicine 17 (4), 315-349 (1995)

[4] Podniesiński, D., Zając, A., „Wybrane aspekty automatyzacji procedur sterowania w laseroterapii”, XIX Krajowa Szkoła Optoelektroniki „Współczesna optoelektronika w medycynie” (in Polish), (2005).

[5] Podniesiński, D., Zając, A., Kęcik, D., „Opracowanie i weryfikacja możliwości wykorzystania układów optycznego sprzężenia zwrotnego w laserowym sprzęcie medycznym przeznaczonym do „spawania” tkanek w wybranych zabiegach laserowych w okulistyce", grant MNiSW Nr 3 T11E 05726 (in Polish), (2006). 
[6] Podniesiński, D., "Automatisation of Steering Procedures on Laser Source Parameters in Medical Equipment", $\mathrm{PhD}$ Thesis (in Polish), (2003).

[7] Zajac, A., Podniesinski, D., Kecik, D., Kecik, M., and Kasprzak, J., "Real-time control procedures for laser welding of biological tissues", Bulletin of the Polish Academy of Sciences Technical Sciences vol. 56 (2), pp 139 - 146 (2008).

[8] Small, V., Celliers, P.M., Da Silva, L.B., and Soltz, B.A. "Two color infrared thermometer for low temperature measurement using a hollow glass optical fiber", Proc. SPIE 2977, 15-20 (1996).

[9] Madura, H., Kastek, M., Piątkowski, T., "Automatic compensation of emissivity in three-wavelength pyrometers", Infrared Physics \& Technology vol. 51, 1-8 (2007).

[10] Bielecki, Z., Chrzanowski, K., Matyszkiel, R., Piatkowski, T., Szulim, M., "Infrared pyrometer for temperature measurement of objects of both wavelength- and time-dependent emissivity", Optica Applicata Volume 29 (3), 284-292 (1999).

[11] Uoon, G., Mordon, S., Brunetaud, J.M., Faiz, R., Straight, R., Bugnon, P.Y., Staroswiecki, M. and Leroux, J. ,"Ono time control of laser pulseses for pseudoconstans temperature coagulation in tissue", Proc. SPIE 1202, 236244 (1990).

[12] Rossi, F., Pini, R., Menabuoni, L., Mencucci, R., Menchini, U., Ambrosini, S., and Vannelli, G.,"Experimental study on the healing process following laser welding of the cornea", J. Biomedical Optics 10 (2), 24004 (2005)

[13] Zając, A., "Chosen Processes of Energy Conversion at Constant Interaction and Impulse Laser Radiation on Biological Tissues", DS thesis (in Polish), (1998).

[14] Zając, A., Polakowski, H., Piatkowski, T., Kastek, M., "Measurements of Tissue Temperatures During Semiconductor Laser Welding Process", Symposium on Photonics and Optoelectronics SOPO (in print), (2012).

[15] Dulski, R., Sosnowski, T., Kastek, M., Trzaskawka, P., "Enhancing image quality produced by IR cameras" Proc. of SPIE Vol. 7834, 783415, (2010). 